\title{
The Minimal Length and the Shannon Entropic Uncertainty Relation
}

\author{
Pouria Pedram \\ Department of Physics, Islamic Azad University, Science and Research Branch, Tehran 1477893855, Iran \\ Correspondence should be addressed to Pouria Pedram; p.pedram@srbiau.ac.ir
}

Received 5 January 2016; Accepted 13 March 2016

Academic Editor: Barun Majumder

Copyright ( 2016 Pouria Pedram. This is an open access article distributed under the Creative Commons Attribution License, which permits unrestricted use, distribution, and reproduction in any medium, provided the original work is properly cited. The publication of this article was funded by SCOAP S $^{3}$

\begin{abstract}
In the framework of the generalized uncertainty principle, the position and momentum operators obey the modified commutation relation $[X, P]=i \hbar\left(1+\beta P^{2}\right)$, where $\beta$ is the deformation parameter. Since the validity of the uncertainty relation for the Shannon entropies proposed by Beckner, Bialynicki-Birula, and Mycielski (BBM) depends on both the algebra and the used representation, we show that using the formally self-adjoint representation, that is, $X=x$ and $P=\tan (\sqrt{\beta} p) / \sqrt{\beta}$, where $[x, p]=i \hbar$, the BBM inequality is still valid in the form $S_{x}+S_{p} \geq 1+\ln \pi$ as well as in ordinary quantum mechanics. We explicitly indicate this result for the harmonic oscillator in the presence of the minimal length.
\end{abstract}

\section{Introduction}

The existence of a minimal observable length proportional to the Planck length $\ell_{\mathrm{P}}=\sqrt{\mathrm{G} \hbar / \mathrm{c}^{3}} \approx 10^{-35} \mathrm{~m}$ is motivated by various proposals of quantum gravity such as string theory, loop quantum gravity, noncommutative geometry, and blackhole [1-3]. Indeed, several schemes have been established to investigate the effects of the minimal length range from astronomical observations $[4,5]$ to table-top experiments [6]. In particular, a measurement method is proposed recently to detect this fundamental length scale which is based on the possible deviations from ordinary quantum commutation relation at the Planck scale within the current technology [6].

Deformed commutation relations have attracted much attention in recent years and several problems range from classical to quantum mechanical systems have been studied exactly or approximately in the context of the generalized (gravitational) uncertainty principle (GUP). Among these investigations in quantum domain, we can mention the harmonic oscillator [7-9], Coulomb potential [10-12], singular inverse square potential [13], coherent states [14], Dirac oscillator [15], Lamb's shift, Landau levels, tunneling current in scanning tunneling microscope [16], ultracold neutrons in gravitational field [17, 18], Casimir effect [19], relativistic quantum mechanics [20-22], and cosmological problems [23-26]. On the other hand, in the classical domain, deformed classical systems in phase space [27, 28], Keplerian orbits [29], composite systems [30], and the thermostatistics $[31,32]$ have been investigated in the presence of the minimal length.

In the last decade, many applications of information theoretic measures such as entropic uncertainty relations, as alternatives to Heisenberg uncertainty relation, appeared in various quantum mechanical systems [33-48]. The concept of statistical complexity was introduced by Shannon in 1948 [49], where the term uncertainty can be considered as a measure of the missing information. In particular, it is shown that the information entropies such as Shannon entropy may be used to replace the well-known quantum mechanical uncertainty relation. The first entropic uncertainty relation for position and momentum observables was proposed by Hirschmann [50] and is later improved by Beckner, Bialynicki-Birula, and Mycielski (BBM) [51-54].

In the context of the generalized uncertainty principle, there exists two questions. (i) Are the wave functions in position space and momentum space related by the Fourier transform in arbitrary GUP algebra in the form $[X, P]=$ $i \hbar f(X, P)$ ? (ii) If in a particular algebra these wave functions 
are not related by the Fourier transform, is the BBM inequality still valid? Note that the validity of the BBM entropic uncertainty relation depends on both the algebra and the used representation. For instance, consider the modified commutation relation in the form $[X, P]=i \hbar\left(1+\beta P^{2}+\alpha X^{2}\right)$ which implies a minimal length and a minimal momentum proportional to $\hbar \sqrt{\beta}$ and $\hbar \sqrt{\alpha}$, respectively. This form of GUP has no formally self-adjoint representation in the form $X=x$ and $P=f(p)$. So the momentum space and coordinate space wave functions are not related by the Fourier transform in any representation and the BBM uncertainty relation does not hold in this framework.

The well-known Robertson uncertainty principle for two noncommuting observables is given by

$$
\Delta A \Delta B \geq \frac{1}{2}|\langle\psi|[A, B]| \psi\rangle|,
$$

where $[A, B]$ denotes the commutator of $A$ and $B$ and $\Delta A$ and $\Delta B$ are their dispersions. However, this uncertainty relation suffers from two serious shortcomings [55, 56]. (i) For two noncommuting observables of a finite $N$-dimensional Hilbert space, since the right-hand side of (1) depends on the wave function $\psi$, it is not a fixed lower bound. Indeed, if $\psi$ is the eigenstate of the observable $A$ or $B$, the righthand side of (1) vanishes and there is no restriction on $\Delta A$ or $\Delta B$ by this uncertainty relation. (ii) The dispersions cannot be considered as suitable measures for the uncertainty of two complementary observables with continuous probability densities. This problem is more notable when their corresponding probability densities contain several sharp peaks. Among various proposals for the uncertainty relations that are not suffered from these shortcomings, we can mention the information-theoretical entropy instead of the dispersions which is a proper measure of the uncertainty.

In this paper, we study the effects of the minimal length on the entropic uncertainty relation. In this scenario, the position and momentum operators obey the modified commutation relation $[X, P]=i \hbar\left(1+\beta P^{2}\right)$, where $\beta$ is the deformation parameter. Using formally self-adjoint representation of the algebra, we show that the coordinate space and momentum space wave functions are related by the Fourier transform and consequently the BBM inequality is preserved. However, as we will show, in the quasiposition representation the momentum space and quasiposition space wave functions are not related by the Fourier transformation and the BBM inequality does not hold. As an application, we obtain the generalized Schrödinger equation for the harmonic oscillator and exactly solve the corresponding differential equation in momentum space. Then, we find information entropies for the two lowest energy eigenstates and explicitly show the validity of the BMM inequality in the presence of the minimal length.

\section{The Generalized Uncertainty Principle}

In one-dimension, the deformed commutation relation reads [7]

$$
[X, P]=i \hbar\left(1+\beta P^{2}\right)
$$

which results in $\Delta X \Delta P \geq(\hbar / 2)\left[1+\beta(\Delta P)^{2}\right]$ (generalized uncertainty principle) and for $\beta \rightarrow 0$ the well-known commutation relation in ordinary quantum mechanics is recovered. Notice that, $\Delta X$ cannot take arbitrarily small values and the absolutely smallest uncertainty in position is given by $(\Delta X)_{\min }=\hbar \sqrt{\beta}$.

Now, consider the formally self-adjoint representation [9]

$$
\begin{aligned}
& X=x, \\
& P=\frac{\tan (\sqrt{\beta} p)}{\sqrt{\beta}},
\end{aligned}
$$

where $[x, p]=i \hbar,-\pi / 2 \sqrt{\beta}<p<\pi / 2 \sqrt{\beta}$, and it exactly satisfies (2). In this representation, the ordinary nature of the position operator is preserved and the inner product of states takes the following form:

$$
\langle\psi \mid \phi\rangle=\int_{-\pi / 2 \sqrt{\beta}}^{+\pi / 2 \sqrt{\beta}} \psi^{*}(p) \phi(p) d p .
$$

Note that although the position operator obeys $X^{\dagger}=$ $X=i \hbar \partial / \partial p$ in momentum space, we have $\mathscr{D}(X) \subset \mathscr{D}\left(X^{\dagger}\right)$. So $X$ is merely symmetric and it is not a true self-adjoint operator. However, based on the von Neumann's theorem, for the momentum operator we obtain $P=P^{\dagger}$ and $\mathscr{D}(P)=$ $\mathscr{D}\left(P^{\dagger}\right)=\left\{\phi \in \mathscr{D}_{\text {max }}(\mathbb{R})\right\}$ [9]. Thus, $P$ is indeed a self-adjoint operator. Moreover, the scalar product and the completeness relation read

$$
\begin{aligned}
\left\langle p^{\prime} \mid p\right\rangle & =\delta\left(p-p^{\prime}\right), \\
\int_{-\pi / 2 \sqrt{\beta}}^{+\pi / 2 \sqrt{\beta}}|p\rangle\langle p| d p & =1 .
\end{aligned}
$$

In momentum space, the eigenfunctions of the position operator are given by the solutions of the eigenvalue equation

$$
X u_{x}(p)=x u_{x}(p)
$$

Here, $u_{x}(p)=\langle p \mid x\rangle$ which can be expressed as

$$
u_{x}(p)=\frac{1}{\sqrt{2 \pi}} \exp \left(-\frac{i p}{\hbar} x\right)
$$

Now, using (6) and (8), coordinate space wave function can be written as

$$
\psi(x)=\frac{1}{\sqrt{2 \pi}} \int_{-\pi / 2 \sqrt{\beta}}^{+\pi / 2 \sqrt{\beta}} e^{i p x / \hbar} \phi(p) d p .
$$

Moreover, $\phi(p)$ is given by the inverse Fourier transform of the coordinate space wave function; namely,

$$
\phi(p)=\frac{1}{\sqrt{2 \pi}} \int_{-\infty}^{+\infty} e^{-i p x / \hbar} \psi(x) d x .
$$

To this end, by taking $\phi(p)=0$ for $|p|>\pi / 2 \sqrt{\beta}$ we can formally extend the domain of the momentum integral (9) to $-\infty<p<\infty$ without changing the coordinate space 
wave function $\psi(x)$. Therefore, $\psi(x)$ is the Fourier transform of $\phi(p)$. Now, using the Babenko-Beckner inequality $[51,52]$ and following Bialynicki-Birula and Mycielski [53] we obtain (see [54] for details)

$$
S_{x}+S_{p} \geq 1+\ln \pi
$$

where

$$
\begin{aligned}
& S_{x}=-\int_{-\infty}^{+\infty}|\psi(x)|^{2} \ln |\psi(x)|^{2} d x, \\
& S_{p}=-\int_{-\infty}^{+\infty}|\phi(p)|^{2} \ln |\phi(p)|^{2} d p,
\end{aligned}
$$

subject to $\phi(p)=0$ for $|p|>\pi / 2 \sqrt{\beta}$. Note that, in this representation, the expression for the entropic uncertainty relation is similar to the ordinary quantum mechanics. However, as we will see in the next section, the presence of the minimal length modifies the Hamiltonian, its solutions, and the values of $S_{x}$ and $S_{p}$. But since $\psi(x)$ and $\phi(p)$ are still related by the Fourier transform, the lower bound for the entropic uncertainty relation will not be modified.

\section{Quasiposition Representation}

Another possible representation that exactly satisfies (2) is [7]

$$
\begin{aligned}
& X \phi(p)=i \hbar\left(1+\beta p^{2}\right) \partial_{p} \phi(p), \\
& P \phi(p)=p \phi(p) .
\end{aligned}
$$

The corresponding scalar product and completeness relations read

$$
\begin{aligned}
\left\langle p^{\prime} \mid p\right\rangle & =\left(1+\beta p^{2}\right) \delta\left(p-p^{\prime}\right), \\
\int_{-\infty}^{+\infty} \frac{1}{1+\beta p^{2}}|p\rangle\langle p| d p & =1 .
\end{aligned}
$$

Now, since the measure in the integral (15) is not flat, the momentum space entropy

$$
S_{p}=-\int_{-\infty}^{+\infty} \frac{1}{1+\beta p^{2}}|\phi(p)|^{2} \ln |\phi(p)|^{2} d p,
$$

has no proper form of the continuous Shannon entropy relation in this representation.

The quasiposition wave function is defined as [7]

$$
\phi(\xi) \equiv\left\langle\psi_{\xi}^{m l} \mid \phi\right\rangle,
$$

where

$$
\psi_{\xi}^{m l}(p)=\sqrt{\frac{2 \sqrt{\beta}}{\pi}}\left(1+\beta p^{2}\right)^{-1 / 2} e^{-i\left(\xi \tan ^{-1}(\sqrt{\beta} p) / \hbar \sqrt{\beta}\right)}
$$

denotes the maximal localization states. These states satisfy $\left\langle\psi_{\xi}^{m l}|X| \psi_{\xi}^{m l}\right\rangle=\xi$ and $(\Delta X)_{\left|\psi_{\xi}^{m l}\right\rangle}=\hbar \sqrt{\beta}$ and are not mutually orthogonal; that is, $\left\langle\psi_{\xi^{\prime}}^{m l} \mid \psi_{\xi}^{m l}\right\rangle \neq \delta\left(\xi-\xi^{\prime}\right)$. In this representation, the relation between the momentum space wave functions and quasiposition wave functions is given by

$\psi(\xi)$

$$
=\sqrt{\frac{2 \sqrt{\beta}}{\pi}} \int_{-\infty}^{+\infty} \frac{1}{\left(1+\beta p^{2}\right)^{3 / 2}} e^{i \xi \tan ^{-1}(\sqrt{\beta} p) / \hbar \sqrt{\beta}} \psi(p) d p .
$$

Thus, the quasiposition wave functions are not Fourier transform of the momentum space wave functions and the quasiposition entropy

$$
\begin{aligned}
S_{\xi} & =-\left(8 \pi \hbar^{2} \sqrt{\beta}\right)^{-1} \\
& \cdot \iiint_{-\infty}^{+\infty} e^{i\left(\xi-\xi^{\prime}\right)\left(\tan ^{-1}(\sqrt{\beta} p) / \hbar \sqrt{\beta}\right)} \psi^{*}(\xi) \psi\left(\xi^{\prime}\right) \\
& \cdot \ln \left|\psi\left(\xi^{\prime}\right)\right|^{2} d p d \xi d \xi^{\prime}
\end{aligned}
$$

does not represent the continuous Shannon entropy and contains plenty of overcounting (because of the nonorthogonality of $\left|\psi_{\xi}^{m l}\right\rangle$ ) that should be avoided. These results show that the information entropies $S_{p}(16)$ and $S_{\xi}(20)$ are not proper measures of uncertainty. However, the information entropies (12) based on formally self-adjoint representation do not suffer from these shortcomings and they can be considered as proper measures of uncertainty in the presence of the minimal length.

\section{Quantum Oscillator}

The Hamiltonian of the harmonic oscillator is given by $H=P^{2} / 2 m+(1 / 2) m \omega^{2} X^{2}$. So the generalized Schrödinger equation in momentum space using the representation (3) reads

$$
-\frac{1}{2} m \hbar^{2} \omega^{2} \frac{d^{2} \phi(p)}{d p^{2}}+\frac{\tan ^{2}(\sqrt{\beta} p)}{2 m \beta} \phi(p)=E \phi(p) .
$$

Using the new variable $\xi=\sqrt{\beta} p$, the above equation can be written as

$$
\frac{d^{2} \phi(\xi)}{d \xi^{2}}+\left(\epsilon-\frac{V}{\cos ^{2} \xi}\right) \phi(\xi)=0,
$$

where $V=(m \beta \hbar \omega)^{-2}$ and $\epsilon=V(1+2 m \beta E)$. Now, taking

$$
\phi_{n}(\xi)=P_{n}(s) \cos ^{\lambda} \xi
$$

results in

$$
\begin{gathered}
\left(1-s^{2}\right) \frac{d^{2} P_{n}(s)}{d s^{2}}-s(1+2 \lambda) \frac{d P_{n}(s)}{d s} \\
+\left(\epsilon-\lambda^{2}\right) P_{n}(s)=0,
\end{gathered}
$$

where $s=\sin \xi$ and

$$
\begin{aligned}
& V=\lambda(\lambda-1), \\
& \lambda=\frac{1}{2}\left[1+\sqrt{1+\frac{4}{m^{2} \beta^{2} \hbar^{2} \omega^{2}}}\right] .
\end{aligned}
$$


It is known that the solutions of the above equation for $\epsilon=$ $(n+\lambda)^{2}$ are given by the Gegenbauer polynomials $C_{n}^{\lambda}(s)$. Therefore, the exact solutions read

$$
\begin{aligned}
\phi_{n}(p) & =N_{n} C_{n}^{\lambda}(\sin (\sqrt{\beta} p)) \cos ^{\lambda}(\sqrt{\beta} p), \\
E_{n} & =\hbar \omega\left(n+\frac{1}{2}\right)\left(\sqrt{1+\frac{\eta^{2}}{4}}+\frac{\eta}{2}\right)+\frac{1}{2} \hbar \omega \eta n^{2}, \\
n & =0,1,2, \ldots,
\end{aligned}
$$

where $\eta=m \beta \hbar \omega, N_{n}$ is the normalization coefficient, and the Gegenbauer polynomials are defined as [57]

$$
C_{n}^{\lambda}(s)=\sum_{k=0}^{[n / 2]}(-1)^{k} \frac{\Gamma(n-k+\lambda)}{\Gamma(\lambda) k !(n-2 k) !}(2 s)^{n-2 k} .
$$

Note that for $\beta \rightarrow 0$ we obtain the ordinary energy spectrum of the harmonic oscillator; that is, $E_{n}=\hbar \omega(n+1 / 2)$. The Gegenbauer polynomials also satisfy the following useful formula [57]:

$$
\begin{array}{r}
\int_{-1}^{1}\left(1-x^{2}\right)^{\nu-1 / 2}\left[C_{n}^{\nu}(x)\right]^{2} d x=\frac{\pi 2^{1-2 \nu} \Gamma(n+2 \nu)}{n !(n+\nu)[\Gamma(\nu)]^{2}} \\
\operatorname{Re} \nu>-\frac{1}{2} .
\end{array}
$$

Since $s=\sin \xi$ and $N_{n}$ is given by the normalization condition $\int_{-\pi / 2 \sqrt{\beta}}^{+\pi / 2 \sqrt{\beta}}|\phi(\xi)|^{2} d p=1$, we find

$$
N_{n}=\sqrt{\frac{\sqrt{\beta} n !(n+\lambda)[\Gamma(\lambda)]^{2}}{\pi 2^{1-2 \lambda} \Gamma(n+2 \lambda)}} .
$$

The solutions can be also written in terms of relativistic Hermite polynomials using the relation [58]

$$
H_{n}^{\lambda}(\sqrt{\lambda} u)=\frac{n !}{\lambda^{n / 2}}\left(1+u^{2}\right)^{n / 2} C_{n}^{\lambda}\left(\frac{u}{\sqrt{1+u^{2}}}\right)
$$

where $H_{n}^{\lambda}(z)$ denotes relativistic Hermite polynomials. Thus, we obtain $C_{n}^{\lambda}(\sin (\sqrt{\beta} p))=\left(\lambda^{n / 2} /\right.$ $n !) \cos ^{n}(\sqrt{\beta} p) H_{n}^{\lambda}(\sqrt{\lambda} \tan (\sqrt{\beta} p))$ which results in

$$
\begin{aligned}
\phi_{n} & (p) \\
& =\frac{N_{n} \lambda^{n / 2}}{n !} \cos ^{\lambda+n}(\sqrt{\beta} p) H_{n}^{\lambda}(\sqrt{\lambda} \tan (\sqrt{\beta} p)) .
\end{aligned}
$$

For the small values of the deformation parameter we have $(m=\hbar=1)$

$$
\begin{aligned}
\lim _{\beta \rightarrow 0} \lambda & =\frac{1}{\omega \beta}, \quad \beta \longrightarrow 0 \Longleftrightarrow \lambda \longrightarrow \infty, \\
\lim _{\lambda \rightarrow \infty} \cos ^{\lambda+n}(\sqrt{\beta} p) & =\exp \left(-\frac{p^{2}}{2 \omega}\right), \\
\lim _{\lambda \rightarrow \infty} \sqrt{\lambda} \tan (\sqrt{\beta} p) & =\frac{p}{\sqrt{\omega}}, \\
\lim _{\lambda \rightarrow \infty} H_{n}^{\lambda}(z) & =H_{n}(z), \quad \text { Ref. [58] }
\end{aligned}
$$

where $H_{n}$ denotes Hermite polynomials. So in this limit the solutions read

$$
\lim _{\beta \rightarrow 0} \phi_{n}(p)=\frac{e^{-p^{2} / 2 \omega} H_{n}(p / \sqrt{\omega})}{\sqrt{2^{n} n ! \sqrt{\pi \omega}}},
$$

which are normalized eigenstates of the ordinary harmonic oscillator as we have expected.

\section{Information Entropy}

The information entropies for the position and momentum spaces can be now calculated for the harmonic oscillator in the GUP framework using (12). In ordinary quantum mechanics and in the position space, the information entropy can be obtained analytically for some quantum mechanical systems. However, since the momentum wave functions are derived from the Fourier transform, the corresponding momentum information entropies are rather difficult to obtain. For our case, as we will show, we find $S_{p}$ analytically for the two lowest energy states and obtain $S_{x}$ numerically. Thus, because of the difficulty in calculating $S_{x}$ and $S_{p}$, we only consider the two lowest energy eigenstates.

First consider the Fourier transform of the momentum space ground state (26) which gives the following state in the position space $(\hbar=1)$ :

$$
\begin{aligned}
& \psi_{0}(x)=\sqrt{\frac{\lambda[\Gamma(\lambda)]^{2}}{\pi^{2} \sqrt{\beta} \Gamma(2 \lambda)}} \frac{\sin [(\pi / 2)(x / \sqrt{\beta}-\lambda)]}{x / \sqrt{\beta}-\lambda} \\
& \cdot{ }_{2} F_{1}\left[\frac{1}{2}\left(\frac{x}{\sqrt{\beta}}-\lambda\right),\right. \\
&\left.-\lambda, \frac{1}{2}\left(\frac{x}{\sqrt{\beta}}-(\lambda-2)\right), 1\right] .
\end{aligned}
$$

Also, for the first excited state $(n=1)$ we have

$$
\begin{aligned}
& \psi_{1}(x) \\
& =i \lambda \sqrt{\frac{(\lambda+1)[\Gamma(\lambda)]^{2}}{\pi^{2} \sqrt{\beta} \Gamma(2 \lambda+1)}}\left\{\frac{\sin [(\pi / 2)(x / \sqrt{\beta}-(\lambda+1))]}{x / \sqrt{\beta}-(\lambda+1)}\right. \\
& \cdot{ }_{2} F_{1}\left[\frac{1}{2}\left(\frac{x}{\sqrt{\beta}}-(\lambda+1)\right),\right. \\
& \left.-\lambda, \frac{1}{2}\left(\frac{x}{\sqrt{\beta}}-(\lambda-1)\right), 1\right] \\
& -\frac{\sin [(\pi / 2)(x / \sqrt{\beta}-(\lambda-1))]}{x / \sqrt{\beta}-(\lambda-1)} \\
& \cdot{ }_{2} F_{1}\left[\frac{1}{2}\left(\frac{x}{\sqrt{\beta}}-(\lambda-1)\right),\right. \\
& \left.\left.-\lambda, \frac{1}{2}\left(\frac{x}{\sqrt{\beta}}-(\lambda-3)\right), 1\right]\right\} .
\end{aligned}
$$

Figure 1 shows the resulting ground state and first excited state in position space for $\beta=\{0,0.5,1\}$. Notice that, 

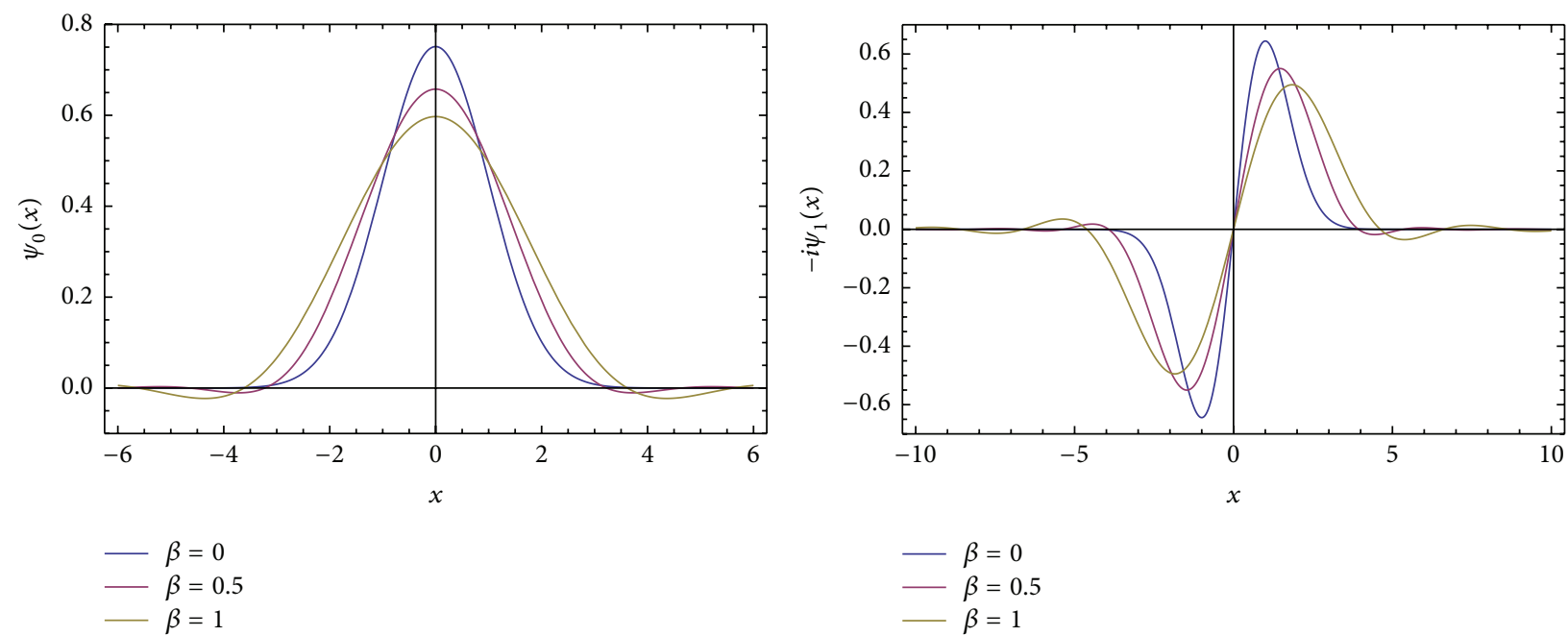

FIGURE 1: Plots of the position space wave functions for $m=\hbar=\omega=1$ and $n=0,1$.
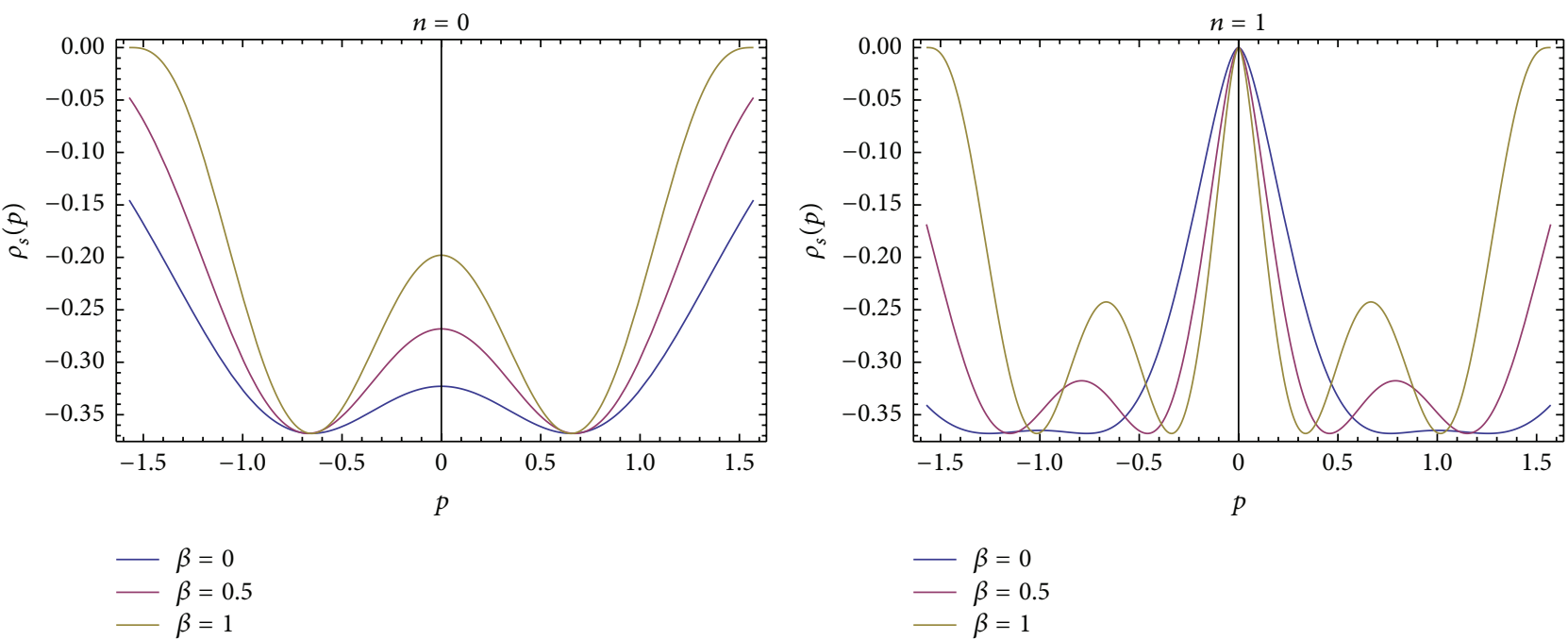

FIgURE 2: Plots of the momentum space entropy densities for $m=\hbar=\omega=1$ and $n=0,1$.

for $\beta \rightarrow 0$, the solutions tend to the simple harmonic oscillator wave functions; that is, $\lim _{\beta \rightarrow 0} \psi_{n}(x)=$ $\sqrt{\sqrt{\omega} / 2^{n} n ! \sqrt{\pi}} e^{-\omega x^{2} / 2} H_{n}(\sqrt{\omega} x)$.

For the ground state, the analytical expression for $S_{p}$ reads

$$
S_{p}^{0}=\lambda H_{\lambda}-\lambda H_{\lambda-1 / 2}+\ln \sqrt{\pi}-\ln \left(\frac{\sqrt{\beta} \lambda \Gamma(\lambda)}{\Gamma(\lambda+1 / 2)}\right)
$$

where $H_{\lambda}$ denotes the harmonic number $H_{n}=\sum_{k=1}^{n} 1 / k$. It is easy to check that, for the small values of $\beta$, the momentum information entropy tends to the ordinary harmonic oscillator information entropy; namely,

$$
\lim _{\beta \rightarrow 0} S_{p}^{0}=\lim _{\beta \rightarrow 0} S_{x}^{0}=\frac{1}{2}(1+\ln \pi) .
$$

For the first excited state, $S_{p}$ is given by

$$
\begin{aligned}
S_{p}^{1}= & (1+\lambda) H_{\lambda+1}-\lambda H_{\lambda-1 / 2}+\ln \sqrt{\pi}-2 \\
& -\ln \left(\frac{\sqrt{\beta} \Gamma(\lambda+2)}{2 \Gamma(\lambda+1 / 2)}\right),
\end{aligned}
$$

and for $\beta \rightarrow 0$ it reads

$$
\lim _{\beta \rightarrow 0} S_{p}^{1}=\lim _{\beta \rightarrow 0} S_{x}^{1}=\frac{1}{2} \ln \pi+\ln 2+\gamma-\frac{1}{2},
$$

where $\gamma \approx 0.5772$ is the Euler constant.

The information entropy densities are defined as $\rho_{s}(x)=$ $|\psi(x)|^{2} \ln |\psi(x)|^{2}$ and $\rho_{s}(p)=|\phi(p)|^{2} \ln |\phi(p)|^{2}$. The behavior of $\rho_{s}(x)$ and $\rho_{s}(p)$ is illustrated in Figures 2 and 3 for $n=$ 0,1 and several values of the deformation parameter. Now, using the numerical values for $S_{x}$, we obtain the left-hand 

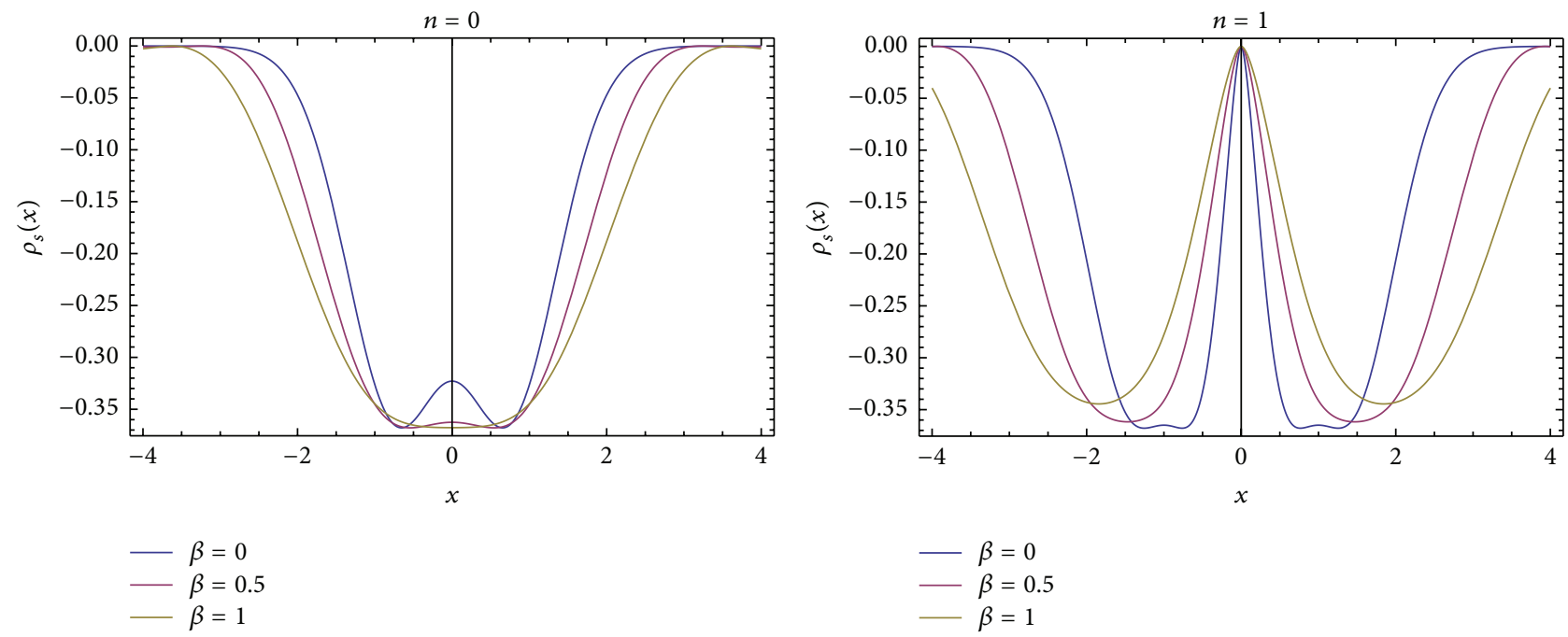

FIgURE 3: Plots of the position space entropy densities for $m=\hbar=\omega=1$ and $n=0,1$.

TABLE 1: The numerical results that establish the BBM entropic uncertainty relation for $m=\hbar=\omega=1$ and various values of $\beta$.

\begin{tabular}{cccccc}
\hline$n$ & $\beta$ & $S_{x}$ & $S_{p}$ & $S_{x}+S_{p}$ & $1+\ln \pi$ \\
\hline \multirow{3}{*}{0} & 0.1 & 1.12153 & 1.02361 & 2.14515 & 2.14473 \\
& 0.5 & 1.30251 & 0.85220 & 2.15471 & 2.14473 \\
& 1.0 & 1.49095 & 0.68153 & 2.17248 & 2.14473 \\
\hline \multirow{3}{*}{1} & 0.1 & 1.40656 & 1.24992 & 2.65648 & 2.14473 \\
& 0.5 & 1.62672 & 0.97566 & 2.60238 & 2.14473 \\
& 1.0 & 1.84423 & 0.74892 & 2.59315 & 2.14473 \\
\hline
\end{tabular}

side of (11). In Table 1, we have reported the position and momentum space information entropies for $\beta=\{0.1,0.5,1\}$ and showed that they obey the BBM inequality. These results indicate that the position space information entropy increases with the GUP parameter $\beta$ and vice versa for the momentum space information entropy but their sum stays above the value $1+\ln \pi$.

\section{Conclusions}

In this paper, we studied the Shannon entropic uncertainty relation in the presence of a minimal measurable length proportional to the Planck length. We showed that using the formally self-adjoint representation, since the coordinate space and momentum space wave functions are related by the Fourier transformation, the measure in the information entropic integral is flat and the lower bound that is predicted by the BBM inequality is guaranteed in the GUP framework. It is worth mentioning that the BBM inequality does not hold for all wave functions. In fact, its validity depends on both the deformed algebra and its representation. As we have indicated, this inequality does not hold in quasiposition representation of our algebra $[X, P]=i \hbar\left(1+\beta P^{2}\right)$. As another example, we mentioned the algebra $[X, P]=i \hbar(1+$ $\left.\alpha X^{2}+\beta P^{2}\right)$ that implies both a minimal length and a minimal momentum. Since this algebra has no formally self-adjoint representation in the form $X=x$ and $P=f(p)$, the momentum space and coordinate space wave functions are not related by the Fourier transform and the BBM uncertainty relation is not valid for this form of GUP. For the case of the harmonic oscillator, we exactly solved the generalized Schrödinger equation in momentum space and found the solutions in terms of the Gegenbauer polynomials. Also, for the two lowest energy eigenstates, we obtained the solutions in position space in terms of the hypergeometric functions. Then, the analytical expressions for the information entropies are found in the momentum space with proper limiting values for $\beta \rightarrow 0$. Using the numerical values for the position information entropy, we explicitly showed that the BBM inequality holds for various values of the deformation parameter. To check the validity of the BBM inequality for other potentials, we need to solve the generalized Schrödinger equation which contains higher order differential terms. However, for the small anharmonic potential terms, the perturbation theory can be used to find the approximate solutions. Also, For other types of GUPs, if a formally selfadjoint representation is viable, the BBM inequality is still valid.

\section{Competing Interests}

The author declares that he has no competing interests.

\section{References}

[1] S. Hossenfelder, "Minimal length scale scenarios for quantum gravity, Living Reviews in Relativity, vol. 16, article 2, 2013.

[2] P. Pedram, "A higher order GUP with minimal length uncertainty and maximal momentum," Physics Letters B, vol. 714, no. 2-5, pp. 317-323, 2012.

[3] P. Pedram, "A higher order GUP with minimal length uncertainty and maximal momentum II: applications," Physics Letters $B$, vol. 718, no. 2, pp. 638-645, 2012. 
[4] G. Amelino-Camelia, J. Ellis, N. E. Mavromatos, D. V. Nanopoulos, and S. Sarkar, "Tests of quantum gravity from observations of big gamma-ray bursts," Nature, vol. 393, pp. 763-765, 1998.

[5] U. Jacob and T. Piran, "Neutrinos from gamma-ray bursts as a tool to explore quantum-gravity-induced Lorentz violation," Nature Physics, vol. 3, pp. 87-90, 2007.

[6] I. Pikovski, M. R. Vanner, M. Aspelmeyer, M. S. Kim, and Č. Brukner, "Probing planck-scale physics with quantum optics," Nature Physics, vol. 8, no. 5, pp. 393-397, 2012.

[7] A. Kempf, G. Mangano, and R. B. Mann, "Hilbert space representation of the minimal length uncertainty relation," Physical Review D, vol. 52, no. 2, pp. 1108-1118, 1995.

[8] L. N. Chang, D. Minic, N. Okamura, and T. Takeuchi, "Exact solution of the harmonic oscillator in arbitrary dimensions with minimal length uncertainty relations," Physical Review D, vol. 65, no. 12, Article ID 125027, 8 pages, 2002.

[9] P. Pedram, "New approach to nonperturbative quantum mechanics with minimal length uncertainty," Physical Review D, vol. 85, no. 2, Article ID 024016, 12 pages, 2012.

[10] T. V. Fityo, I. O. Vakarchuk, and V. M. Tkachuk, "Onedimensional Coulomb-like problem in deformed space with minimal length," Journal of Physics A: Mathematical and General, vol. 39, no. 9, pp. 2143-2149, 2006.

[11] P. Pedram, "A note on the one-dimensional hydrogen atom with minimal length uncertainty," Journal of Physics A: Mathematical and Theoretical, vol. 45, no. 50, Article ID 505304, 11 pages, 2012.

[12] P. Pedram, "One-dimensional hydrogen atom with minimal length uncertainty and maximal momentum," Europhysics Letters, vol. 101, no. 3, Article ID 30005, 2013.

[13] D. Bouaziz and M. Bawin, "Regularization of the singular inverse square potential in quantum mechanics with a minimal length," Physical Review A, vol. 76, Article ID 032112, 2007.

[14] P. Pedram, "Coherent states in gravitational quantum mechanics," International Journal of Modern Physics D, vol. 22, no. 2, Article ID 1350004, 14 pages, 2013.

[15] C. Quesne and V. M. Tkachuk, "Dirac oscillator with nonzero minimal uncertainty in position," Journal of Physics A. Mathematical and General, vol. 38, no. 8, pp. 1747-1765, 2005.

[16] S. Das and E. C. Vagenas, "Universality of quantum gravity corrections," Physical Review Letters, vol. 101, Article ID 221301, 2008.

[17] P. Pedram, K. Nozari, and S. H. Taheri, "The effects of minimal length and maximal momentum on the transition rate of ultra cold neutrons in gravitational field," Journal of High Energy Physics, vol. 2011, no. 3, article 093, 2011.

[18] P. Pedram, "Exact ultra cold neutrons' energy spectrum in gravitational quantum mechanics," The European Physical Journal C, vol. 73, no. 10, article 2609, 2013.

[19] A. M. Frassino and O. Panella, "Casimir effect in minimal length theories based on a generalized uncertainty principle," Physical Review D, vol. 85, no. 4, Article ID 045030, 2012.

[20] P. Pedram, "Dirac particle in gravitational quantum mechanics," Physics Letters B, vol. 702, no. 4, pp. 295-298, 2011.

[21] P. Pedram, "Nonperturbative effects of the minimal length uncertainty on the relativistic quantum mechanics," Physics Letters B, vol. 710, no. 3, pp. 478-485, 2012.

[22] P. Pedram, M. Amirfakhrian, and H. Shababi, "On the $(2+1)-$ dimensional Dirac equation in a constant magnetic field with a minimal length uncertainty," International Journal of Modern Physics D, vol. 24, no. 2, Article ID 1550016, 8 pages, 2015.
[23] P. Pedram, "Generalized uncertainty principle and the conformally coupled scalar field quantum cosmology," Physical Review D, vol. 91, no. 6, Article ID 063517, 10 pages, 2015.

[24] H. R. Sepangi, B. Shakerin, and B. Vakili, "Deformed phase space in a two-dimensional minisuperspace model," Classical and Quantum Gravity, vol. 26, no. 6, Article ID 065003, 21 pages, 2009.

[25] B. Vakili, "Dilaton cosmology, noncommutativity, and generalized uncertainty principle," Physical Review D, vol. 77, no. 4, Article ID 044023, 10 pages, 2008.

[26] S. Jalalzadeh, S. M. M. Rasouli, and P. V. Moniz, "Quantum cosmology, minimal length, and holography," Physical Review D, vol. 90, no. 2, Article ID 023541, 2014.

[27] S. Benczik, L. N. Chang, D. Minic, N. Okamura, S. Rayyan, and T. Takeuchi, "Short distance versus long distance physics: the classical limit of the minimal length uncertainty relation," Physical Review D, vol. 66, no. 2, Article ID 026003, 2002.

[28] A. M. Frydryszak and V. M. Tkachuk, "Aspects of pre-quantum description of deformed theories," Czechoslovak Journal of Physics, vol. 53, no. 11, pp. 1035-1040, 2003.

[29] Z. K. Silagadze, "Quantum gravity, minimum length and Keplerian orbits," Physics Letters A, vol. 373, no. 31, pp. 2643-2645, 2009.

[30] C. Quesne and V. M. Tkachuk, "Composite system in deformed space with minimal length," Physical Review A, vol. 81, no. 1, Article ID 012106, 2010.

[31] B. Vakili and M. A. Gorji, "Thermostatistics with minimal length uncertainty relation," Journal of Statistical Mechanics: Theory and Experiment, vol. 2012, no. 10, Article ID P10013, 2012.

[32] M. Abbasiyan-Motlaq and P. Pedram, "Generalized uncertainty principle and thermostatistics: a semiclassical approach," International Journal of Theoretical Physics, vol. 55, no. 4, pp. 19531961, 2016.

[33] S. Wehner and A. Winter, "Entropic uncertainty relations-a survey," New Journal of Physics, vol. 12, Article ID 025009, 2010.

[34] V. Majernik and T. Opatrny, "Entropic uncertainty relations for a quantum oscillator," Journal of Physics A, vol. 29, no. 9, pp. 2187-2197, 1996.

[35] S. Dong, G.-H. Sun, S.-H. Dong, and J. P. Draayer, "Quantum information entropies for a squared tangent potential well," Physics Letters A, vol. 378, no. 3, pp. 124-130, 2014.

[36] R. Atre, A. Kumar, C. N. Kumar, and P. Panigrahi, "Quantuminformation entropies of the eigenstates and the coherent state of the Pöschl-Teller potential," Physical Review A, vol. 69, no. 5, Article ID 052107, 6 pages, 2004.

[37] E. Romera and F. de los Santos, "Identifying wave-packet fractional revivals by means of information entropy," Physical Review Letters, vol. 99, Article ID 263601, 2007.

[38] J. S. Dehesa, A. Martínez-Finkelshtein, and V. N. Sorokin, "Information-theoretic measures for Morse and Pöschl-Teller potentials," Molecular Physics, vol. 104, no. 4, pp. 613-622, 2006.

[39] E. Aydiner, C. Orta, and R. Sever, "Quantum information entropies of the eigenstates of the morse potential," International Journal of Modern Physics B, vol. 22, no. 3, pp. 231-237, 2008.

[40] A. Kumar, "Information entropy of isospectral Pöschl-Teller potential," Indian Journal of Pure and Applied Physics, vol. 43, no. 12, pp. 958-963, 2005.

[41] J. Katriel and K. D. Sen, "Relativistic effects on information measures for hydrogen-like atoms," Journal of Computational and Applied Mathematics, vol. 233, no. 6, pp. 1399-1415, 2010. 
[42] S. H. Patil and K. D. Sen, "Net information measures for modified Yukawa and Hulthén potentials," International Journal of Quantum Chemistry, vol. 107, no. 9, pp. 1864-1874, 2007.

[43] K. D. Sen, "Characteristic features of Shannon information entropy of confined atoms," The Journal of Chemical Physics, vol. 123, no. 7, Article ID 074110, 2005.

[44] D. Dutta and P. Roy, "Information entropy of conditionally exactly solvable potentials," Journal of Mathematical Physics, vol. 52, no. 3, Article ID 032104, 2011.

[45] H. Grinberg, "Quadrature squeezing and information entropy squeezing in nonlinear two-level spin models," International Journal of Modern Physics B, vol. 24, no. 9, pp. 1079-1092, 2010.

[46] M. Abdel-Aty, I. A. Al-Khayat, and S. S. Hassan, "Shannon information and entropy squeezing of a single-mode cavity QED of a raman interaction," International Journal of Quantum Information, vol. 4, no. 5, pp. 807-814, 2006.

[47] H. G. Laguna and R. P. Sagar, "Shannon entropy of the wigner function and position-momentum correlation in model systems," International Journal of Quantum Information, vol. 8, no. 7, pp. 1089-1100, 2010.

[48] H. H. Corzo, H. G. Laguna, and R. P. Sagar, "Localizationdelocalization phenomena in a cyclic box," Journal of Mathematical Chemistry, vol. 50, no. 1, pp. 233-248, 2012.

[49] C. E. Shannon, "A mathematical theory of communication," The Bell System Technical Journal, vol. 27, no. 4, pp. 623-656, 1948, (Reprinted in: C. E. Shannon, Claude Elwood Shannon: Collected Papers, IEEE Press, New York, NY, USA, 1993).

[50] I. I. Hirschmann, "A note on entropy," American Journal of Mathematics, vol. 79, no. 1, pp. 152-156, 1957.

[51] K. I. Babenko, "An inequality in the theory of Fourier integrals," Izvestiya Rossiiskoi Akademii Nauk, Seriya Matematicheskaya, vol. 25, no. 4, pp. 531-542, 1961.

[52] W. Beckner, "Inequalities in Fourier analysis," Annals of Mathematics, vol. 102, no. 1, pp. 159-182, 1975.

[53] I. Bialynicki-Birula and J. Mycielski, "Uncertainty relations for information entropy in wave mechanics," Communications in Mathematical Physics, vol. 44, no. 2, pp. 129-132, 1975.

[54] I. Bialynicki-Birula and L. Rudnicki, "Entropic uncertainty relations in quantum physics," in Statistical Complexity: Applications in Electronic Structure, pp. 1-34, Springer, Berlin, Germany, 2011.

[55] J. Sánchez-Ruiz, "Maassen-Uffink entropic uncertainty relation for angular momentum observables," Physics Letters A, vol. 181, no. 3, pp. 193-198, 1993.

[56] J. B. M. Uffink and J. Hilgevoord, "Uncertainty principle and uncertainty relations," Foundations of Physics, vol. 15, no. 9, pp. 925-944, 1985.

[57] I. S. Gradshteyn and I. M. Ryzhik, Tables of Integrals, Series and Products, Academic Press, New York, NY, USA, 5th edition, 1994.

[58] B. Nagel, "The relativistic Hermite polynomial is a Gegenbauer polynomial," Journal of Mathematical Physics, vol. 35, no. 4, pp. 1549-1554, 1994. 

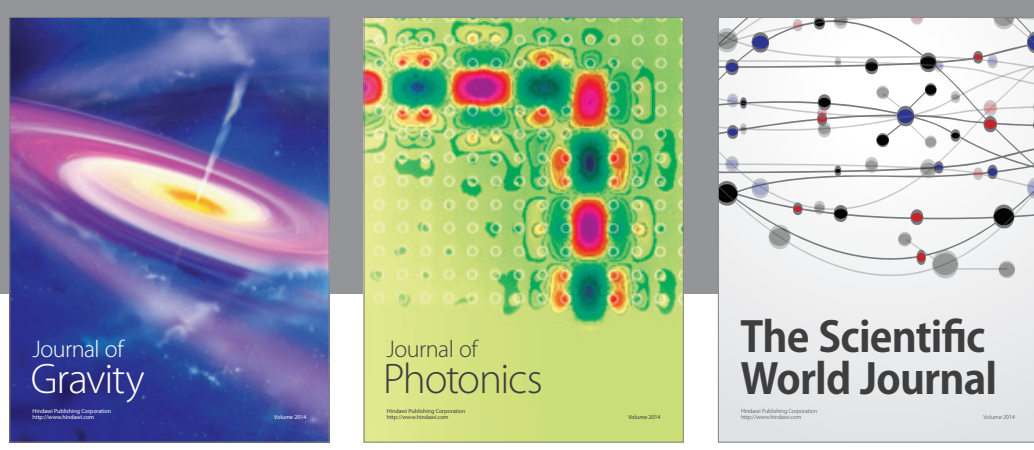

The Scientific World Journal
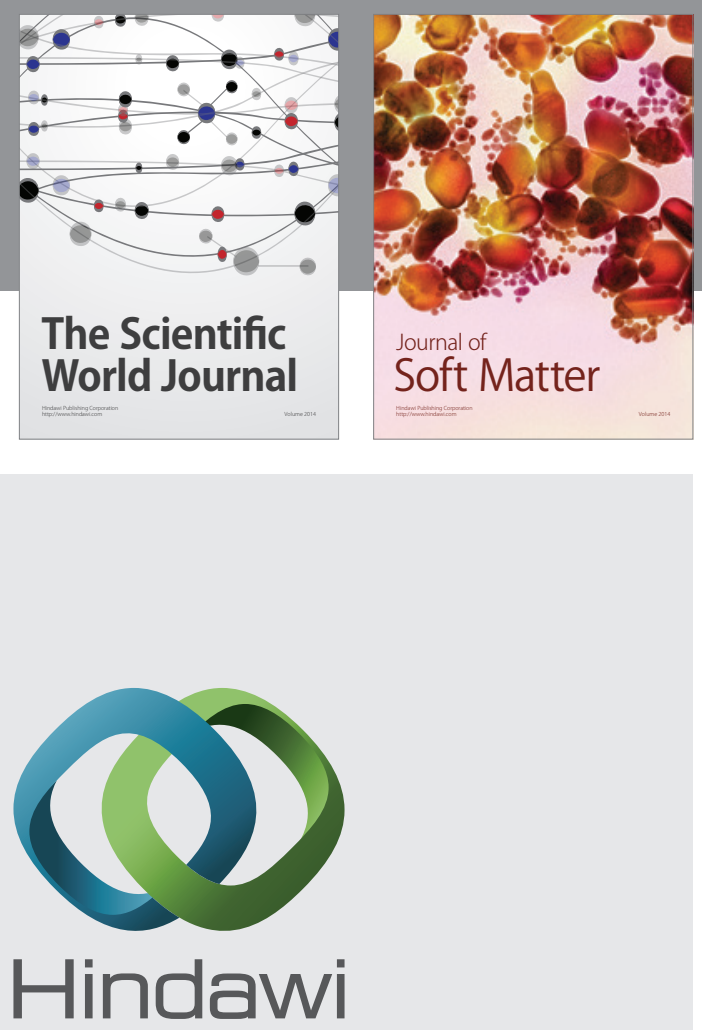

Submit your manuscripts at

http://www.hindawi.com

nternational Journal of

Statistical Mechanics
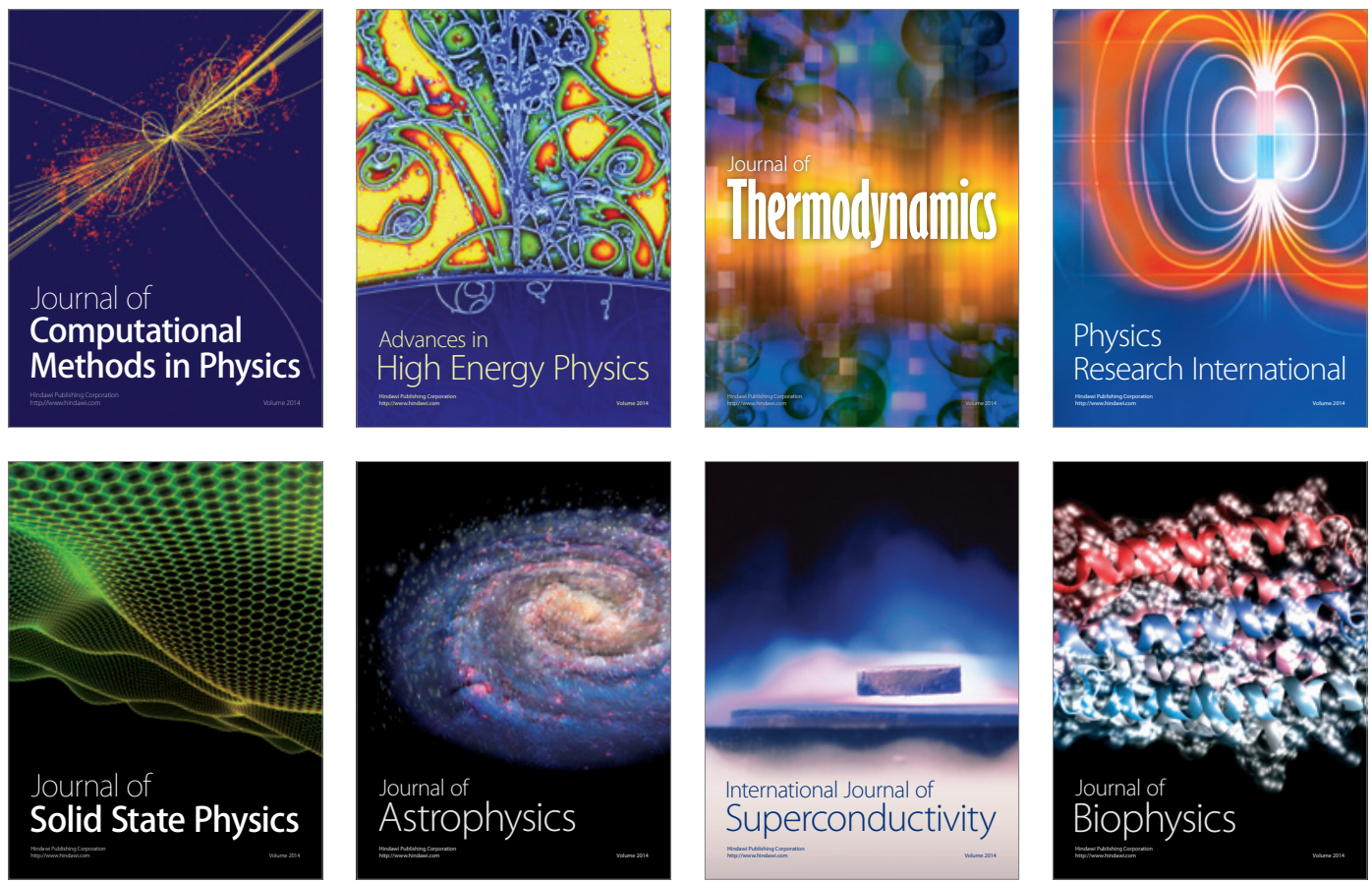
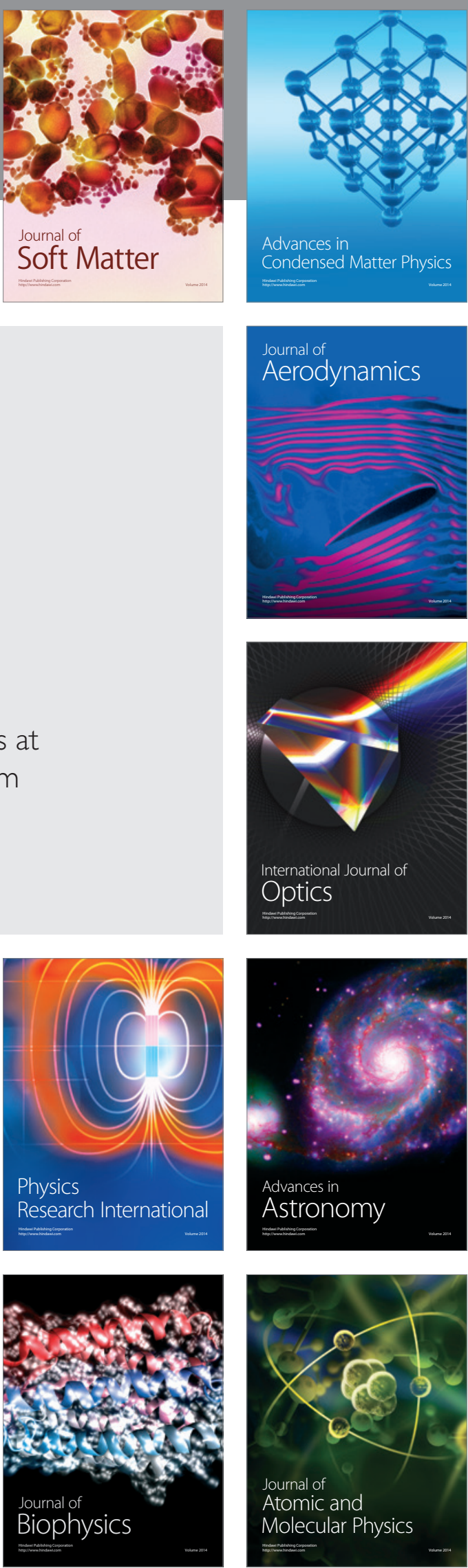